\title{
NOVAS PROPOSTAS PARA O PROJETO DE REGIONALIZAÇÃO DA SAÚDE NO ESTADO DO PARANÁ (SUS-PR) UTILIZANDO O ALGORITMO BRANCH-AND-PRICE
}

\section{A NEW PROPOSAL FOR THE PROJECT OF THE HEALTH SYSTEM IN THE STATE OF PARANÁ (SUS-PR) USING THE ALGORITHM BRANCH- AND-BOUND}

\author{
Cassius Tadeu Scarpin ${ }^{1}$; Maria Teresinha Arns Steiner ${ }^{2}$; Gláucio José Cardozo Dias ${ }^{3}$ \\ ${ }^{1}$ Universidade Federal do Paraná - UFPR - Curitiba - Brasil cassiusts@yahoo.com.br \\ ${ }^{2}$ Universidade Federal do Paraná - UFPR - Curitiba - Brasil tere@ufpr.br \\ ${ }^{3}$ Universidade Federal do Paraná - UFPR - Curitiba - Brasil Brasil glaucio.dias@datasul.com.br
}

\begin{abstract}
Resumo
Neste trabalho são apresentadas três novas propostas para o projeto de regionalização da saúde no estado do Paraná. O projeto consiste em dividir o estado em regiões menores, em três níveis hierárquicos: macro-regiões, regionais e micro-regiões. Cada uma dessas divisões possui uma cidade-sede, sendo esta a principal responsável pelo atendimento médico de sua divisão hierárquica. A formação otimizada de grupos de cidades é o principal objetivo deste trabalho. $O$ problema de otimização está relacionado à definição de p-medianas, em uma rede de $n$ nós, de modo a minimizar a soma das distâncias dos pontos de demanda às p medianas mais próximas. $\mathrm{Na}$ solução deste problema fez-se uso do algoritmo branch-and-price. Além disso, combina-se a relaxação Lagrangeana/surrogate com a geração de colunas para fornecer colunas mais produtivas para encontrar a melhor solução possível. Novas configurações hierárquicas e novas politicas para as cidades-sede são sugeridas neste trabalho. Os resultados mostraram-se bastante satisfatórios.
\end{abstract}

Palavras-chave: Regionalização da Saúde; Problema das p-medianas; Algoritmo Branch-and-Price.

\section{Introdução}

O trabalho da logística é prover disponibilidade de produtos e/ou serviços, onde e quando estes forem necessários de forma otimizada. Uma questão básica do gerenciamento logístico é como estruturar sistemas e/ou configurações de distribuição capazes de atender de forma econômica os mercados geograficamente distantes das fontes de produção, oferecendo níveis de serviço cada vez mais altos em termos de disponibilidade de estoque ou capacidade de atendimento em um intervalo de tempo cada vez menor.

Decisões sobre a melhor configuração para instalação de facilidades/instalações destinadas 
ao atendimento da demanda de uma população são tratadas em uma ampla classe de problemas, conhecidos como Problemas de Localização. O modelo clássico utilizado para representação dos problemas desta classe é o problema das p-medianas, o qual procura em um grafo de n nós, escolher p nós como sendo as facilidades, procurando minimizar a soma das distâncias dos nós de demanda à facilidade mais próxima (ROSÁRIO et al., 2002).

As 399 cidades do estado do Paraná e suas ligações, formam um grafo que pode ser estruturado para a definição das p-medianas, sendo as cidades os pontos do grafo e as estradas entre elas os arestas/arcos. Desta forma, tem-se na regionalização da saúde, uma possibilidade de aplicação da modelagem mencionada.

O projeto de regionalização da saúde é uma proposta que permite organizar as cidades em divisões hierárquicas de forma a facilitar o deslocamento dos pacientes que necessitam de atendimento fora das suas cidades de origem (BRASIL, 2004). Para definir as diversas divisões hierárquicas (micro-regiões, regionais e macro-regiões), o estado utiliza os acordos entre as cidades, chamadas pactuações, onde uma cidade se responsabiliza pelo atendimento aos cidadãos das cidades "pactuadas", originando assim as cidades-sede.

Neste trabalho, propõe-se uma nova forma para definição das cidades-sede, construindo de forma otimizada as divisões hierárquicas, baseando-se no modelo clássico do problema das pmedianas. Propõem-se também novas configurações hierárquicas em relação ao número de divisões. Para a solução do problema utiliza-se o método branch-and-price, que está baseado no tradicional método de branch-and-bound, com a aplicação do método de geração de colunas utilizando a relaxação lagrangeana/surrogate como alternativa de estabilização, em cada nó da árvore.

Um dos objetivos da regionalização da saúde em um estado é o melhoramento do fluxo de pacientes que não encontram o procedimento médico necessário em sua cidade (BRASIL, 2002). Uma metodologia para a definição do fluxo de pacientes foi desenvolvida em Scarpin et al. (2006), que combinada com a otimização da construção das divisões hierárquicas trazem significativas melhorias na utilização e no controle do sistema de saúde no estado do Paraná.

Na seção 2 é apresentado o problema da regionalização da saúde. Na seção 3 é apresentado uma definição para o problema das p-medianas, o modelo matemático utilizado para a geração de colunas e as sub-rotinas utilizadas no algoritmo branch-and-price. Os testes computacionais são apresentados na seção 4 e as conclusões deste trabalho estão na seção 5 . 


\section{A Regionalização da Saúde}

A definição estabelecida pelo Ministério da Saúde, em Brasil (2002), sobre o processo de regionalização da saúde no Brasil diz:

"Estabelecer o processo de regionalização como estratégia de hierarquização dos serviços de saúde e de busca de maior eqüidade: o processo de regionalização deverá contemplar uma lógica de planejamento integrado, compreendendo as noções de territorialidade, na identificação de prioridade de intervenção e de conformação de sistemas funcionais de saúde, não necessariamente restritos à abrangência municipal, mas respeitando seus limites como unidade indivisível, de forma a garantir o acesso dos cidadãos a todas as ações e serviços necessários para resolução de seus problemas de saúde, otimizando os recursos disponíveis".

Deve-se notar que não é definida a forma de realização do planejamento, deixando esta tarefa ao encargo dos próprios municípios. O grande problema encontrado para a regionalização da saúde é a forma de como realizar as pactuações entre cidades, ou seja, de como dividir o estado em regiões que realmente garantam o acesso à saúde para população (OLIVEIRA, 2005).

O processo de regionalização deve contemplar sistemas completos que visem garantir o acesso facilitado do usuário a todos os serviços necessários. A facilidade de acesso significa, principalmente, garantir a menor distância possível entre o usuário e o serviço; além disso, a responsabilidade pela qualidade e pelos encaminhamentos deve ser dos gestores e não dos usuários (PARANÁ, 2001). Há muitos municípios que não conseguem realizar os serviços básicos de atendimento e, com isso, torna-se cada vez mais necessário encontrar as divisões de macro-regiões, regionais e micro-regiões da saúde para possibilitar à população a garantia de acesso ao atendimento dos seus problemas de saúde (RIO GRANDE DO NORTE, 2004).

\section{O Problema das p-medianas}

O problema das $p$-medianas é um problema de localização-alocação que tem por objetivo determinar a solução que forneça o menor custo possível para instalação de facilidades de atendimento de demanda de clientes em uma rede conectada por um número finito de caminhos. $\mathrm{Na}$ grande maioria das vezes, o resultado do problema fornece apenas uma configuração que apresenta o custo mínimo, porém, existem casos onde mais de uma configuração pode resultar na solução ótima.

As características de um problema das $p$-medianas são: apresenta um número $(n)$ finito de pontos/nós/vértices conhecidos como pontos de demanda; um número finito de locais candidatos 
para a instalação de facilidades; a matriz de distâncias entre os $n$ vértices e os locais candidatos, formam um grafo e, por fim, existe um número $p$ de facilidades a serem instaladas. Os locais candidatos para a instalação de facilidades podem ser os próprios $n$ pontos de demanda, que é o caso deste trabalho.

O problema das $p$-medianas também pode ser apresentado da seguinte maneira: particionar o conjunto $N$ em $p$ agrupamentos disjuntos de pontos chamados grupos (clusters) e minimizar a diferença entre os elementos de cada cluster (RAO, 1971).

A formulação do problema das p-medianas utilizando a formulação relaxada de particionamento de conjuntos (PPC), com restrição de cardinalidade, é apresentada a seguir.

$P P C$

$$
\begin{array}{ll} 
& \text { v(PPC })=\operatorname{Min} \sum_{k \in M} c_{k} x_{k} \\
\text { s.a. } \quad & \sum_{k \in M} A^{k} x_{k} \geq 1 \\
& \sum_{k \in M} x_{k}=p \\
& x_{k} \in\{0,1\}, \quad \forall k \in M
\end{array}
$$

onde $M=\{1,2, \ldots, m\}$, sendo $m$ o número de colunas (cada coluna é uma variável) usadas no modelo; $c_{k}=\operatorname{Min}_{j \in S_{k}}\left\{\sum_{i \in S_{k}} d_{i j}\right\}, \forall k \in M ; A^{k}=\left[a_{i k}\right]_{n x l}$, com $a_{i k}=1$ se o vértice $i \in S_{k}, a_{i k}=0$ caso contrário; $x_{k}=1$ se o subconjunto (cluster) $S_{k} \in S$ for escolhido, $x_{k}=0$ caso contrário.

$\mathrm{Na}$ restrição (2) cada coluna $A^{k}$ corresponde a um subconjunto $S_{k}$, representando um cluster cuja mediana $j \in S_{k}$ é determinada quando o custo $c_{k}$ é calculado. O cálculo de $c_{k}, \forall k \in M$, é feito da seguinte forma: o nó $j \in S_{k}$ que corresponder ao menor valor de distância total aos outros nós $i \in S_{k}$ será escolhido como mediana, e o valor da distância total calculada corresponderá ao custo do cluster $k$. As restrições (3) e (4) garantem o número exato de medianas requerida (solicitada) e a integralidade das variáveis respectivamente. Poderá ocorrer que dois ou mais vértices $j \in S_{k}$ correspondam ao menor valor de custo do dado subconjunto, assim como critério de desempate, poderá ser definida como mediana, o vértice de maior índice ou outro como preferir.

A restrição (2) garante que as soluções viáveis para PPC são formadas por combinações de $p$ colunas $A^{k}$ com cada vértice $j \in N$ pertencendo a somente um subconjunto $S_{k}$. A característica que determina a viabilidade das soluções de problemas das $p$-medianas é que cada vértice de demanda seja atendido por apenas uma facilidade, garantida pela restrição (3); deste modo no contexto de problemas de localização a condição é satisfeita e, devido a ela, a obtenção das soluções é dificultada. 
Essa versão relaxada da formulação PPC pode ser utilizada devido aos coeficientes positivos da matriz de distâncias $\left[d_{i j}\right]_{n \times n}$, onde a restrição (2) é transformada na desigualdade mostrada, mantendo a viabilidade das soluções obtidas para o problema das $p$-medianas.

Pode-se verificar a afirmação anterior facilmente ao se considerar que, para uma dada solução viável $x_{k}$, se um vértice está presente em duas colunas simultaneamente, ele pode ser removido de uma delas e o custo resultante da nova coluna será menor que o custo da coluna original, reduzindo assim o valor da função objetivo (1).

\subsection{O Problema Mestre Restrito}

Partindo-se de um conjunto inicial de colunas, novas colunas são inseridas na formulação de um problema mestre, a cada iteração. Esse é o processo iterativo para a resolução de problemas lineares de grande porte pelo método de geração de colunas.

Um problema mestre restrito (PMR) para resolver o problema das $p$-medianas pode ser definido, considerando-se um subconjunto $K \subset M=\{1,2, \ldots, m\}$ dos índices das colunas da formulação completa do problema de particionamento de conjuntos, como a seguinte formulação de relaxação de Programação Linear de um problema de cobertura de conjuntos com restrição de cardinalidade.

$$
\begin{gathered}
v(\overline{P C C})=\operatorname{Min} \sum_{k \in K} c_{k} x_{k} \\
\text { s.a. } \quad \sum_{k \in K} A^{k} x_{k} \geq 1 \\
\sum_{k \in K} x_{p}=p \\
x_{k} \in[0,1] \quad \forall k \in K
\end{gathered}
$$

Observa-se que as variáveis $x_{k}$ podem assumir valores fracionários, entre zero e um e, por esse motivo, a solução de $\overline{P C C}$ pode não ser viável para o $P P C$. A aplicação de métodos tipo branch-and-bound pode produzir soluções viáveis para o $P P C$, mas o conjunto final de colunas do $\overline{P C C}$ pode não ser suficiente para a obtenção de solução ótima. As restrições duais ótimas $\lambda \in R_{+}^{n}$ e $\mu \in R$, associadas às restrições (6) e (7) respectivamente, serão utilizadas para o cálculo de novas colunas.

\subsection{O Algoritmo Branch-and-Price}

O método branch-and-price (NARCISO, 1998) está baseado no método branch-and-bound para obtenção de soluções viáveis para o problema das $p$-medianas, onde em cada nó da árvore de 
busca utiliza-se o método de geração de colunas aplicado ao modelo matemático do problema de particionamento de conjuntos. No algoritmo, os problemas resultantes da aplicação das regras de ramificação, neste trabalho, são armazenados em uma lista, a qual é determinada por prioridades, buscando explorar os nós mais promissores para a obtenção de uma solução ótima.

Ao obter-se uma solução não inteira utiliza-se a técnica de ramificação que diferencia os coeficientes de linha $(q, r)$ que são do mesmo cluster (pertencentes a uma mesma mediana) ou não. Para a identificação do par de vértices $(q, r)$ observa-se somente as colunas que possuírem valores fracionários na linha da função objetivo. Para que a árvore de busca não seja explorada em nós não promissores impõem-se condições de poda para tais nós e assim economiza-se esforço computacional significativo. Uma vez determinados os índices de linha $(q, r)$, são acrescentadas ao problema restrições para que se possa encontrar uma solução final inteira.

\subsubsection{A Geração de Colunas}

Ao reescrever a formulação do problema das $p$-medianas para a formulação do problema de particionamento de conjuntos, procura-se fazer a combinação de todas as possíveis soluções viáveis para o problema. Ao se escolher $p$ subconjuntos de $S=\left\{S_{1}, S_{2}, \ldots, S_{m}\right\}$ que representem uma solução viável para o problema pode-se, dentre várias dessas combinações, encontrar a solução de menor custo que será a solução ótima desejada. As técnicas utilizadas no desenvolvimento do algoritmo são baseadas em Pereira et al. (2005).

A ordem de grandeza do conjunto $S$, pode ser muito grande. Para um problema com $n$ vértices, o conjunto $S$ relativo terá a grandeza da ordem de $2^{\mathrm{n}}$ elementos e a enumeração de todos os subconjuntos $S_{k} \in S$; quando o valor de $n$ vai aumentando, torna-se uma tarefa exponencialmente mais difícil. Como cada subconjunto $S_{k}$ é uma coluna das restrições da formulação para o problema de particionamento de conjuntos, então se escritas as restrições com todos os subconjuntos $S_{k}$, o número de variáveis seria enorme, pois cada coluna está relacionada a uma variável, e a dificuldade computacional seria um desafio extremamente árduo.

Quando é utilizada a técnica de geração de colunas pode-se manipular o conjunto de colunas usadas de acordo com as informações das variáveis duais de um problema mestre. Um conjunto bastante reduzido de colunas é utilizado inicialmente. Novas colunas são calculadas como soluções de subproblemas e somente as novas colunas que contribuírem para a melhoria da solução principal serão utilizadas. Em um problema de minimização (maximização), a coluna de custo reduzido mais negativo (positivo) é adicionada à matriz de restrições, gerando um novo problema mestre, cuja resolução fornecerá uma nova solução dual que será utilizada para modificar os coeficientes de 
custo do subproblema gerador de colunas. Quando não houver mais colunas com custo reduzido negativo (positivo) a serem incluídas na matriz de restrições, o processo é encerrado.

Há, inclusive, uma estratégia chamada de multi-pricing que permite adicionar todas as colunas de custo reduzido negativo (positivo) obtidas na resolução do subproblema gerador de colunas em cada iteração. Essa estratégia visa acelerar o processo de resolução do problema.

\subsubsection{O Conjunto Inicial de Colunas}

A importância de um conjunto inicial de colunas é muito grande quanto ao sucesso ou fracasso na resolução do PMR. Obtendo-se um conjunto de colunas inicial que proporcione viabilidade para o PMR, o desenvolvimento do procedimento de geração de colunas consistirá em melhorar a solução a cada iteração.

Uma rotina desenvolvida por Pereira et al. (2005) sugere a construção desse conjunto da seguinte maneira:

- Defina $M a x C$ como o número máximo de colunas a serem geradas;

- Faça $N u n C \leftarrow 0$, onde $N u n C$ é o número de colunas geradas nas iterações.

- Repita

- Seja $P=\left\{n_{1}, \ldots, n_{p}\right\}$ contido em $N$ um conjunto de vértices escolhido aleatoriamente;

- $\operatorname{Para} j=1, \ldots, p$ faça:

- $S_{j} \leftarrow\left\{n_{j}\right\} \cup\left\{q \in N-P \mid d_{q n_{j}}=\min _{t \in P}\left\{d_{q t}\right\}\right\}$

- $c_{j} \leftarrow \operatorname{Min}_{t \in S_{j}}\left\{\sum_{i \in S_{j}} d_{i t}\right\}$

- $\quad$ Para $i=1, \ldots, n$ faça

- Se $i \in S_{j}$, faça $a_{i j} \leftarrow 1$;

- Se $i \notin S_{j}$, faça $a_{i j} \leftarrow 0$;

- Adicione a coluna $A^{j}$ ao conjunto inicial de colunas;

- $N u n C \leftarrow N u n C+p$;

- Enquanto $(\mathrm{NumC}<\operatorname{Max} C)$;

- Fim

Uma base viável para o problema linear inicial deve estar contida nesse conjunto de colunas inicial. Um novo conjunto de $p$ colunas é adicionado a cada iteração. 


\subsubsection{O Subproblema Gerador de Colunas}

A integração do processo de geração de colunas com a relaxação lagrangeana/surrogate é feita na resolução do subproblema gerador de colunas ao utilizar os multiplicadores duais ótimos $\lambda_{i}, \forall i \in N$, do problema $\overline{P C C}$, para resolver o problema $D_{t, \lambda}$ para encontrar o melhor valor do multiplicador $t$ :

$$
D_{t, \lambda}\left(D_{t, \lambda}\right)=\operatorname{Max}_{t}\left\{v\left(L_{t} S_{\lambda} P P M\right)\right\}
$$

onde para encontrá-lo necessita-se resolver:

$$
\begin{aligned}
& v\left(L_{t} S_{\lambda} P P M\right)=\sum_{j \in N} \beta_{j} x_{j j}^{\lambda}+t \sum_{i \in N} \lambda_{i} \text {, sendo } \\
& \beta_{j}=\sum_{i \in N} \min \left\{0, d_{i j}-t \lambda_{i}\right\}, \quad \forall j \in N
\end{aligned}
$$

e definindo-se $J$ como o conjunto dos índices $j \in N$ que correspondem aos $p$ menores valores de $\beta_{j}$. Assim , uma solução $x^{\lambda}$ para $L_{t} S_{\lambda} P P M$ pode ser obtida fazendo-se:

$x_{j j}^{\lambda}=\left\{\begin{array}{ll}1, & \text { se } j \in J \\ 0, & \text { caso contrário }\end{array}\right.$ e para $i \neq j: x_{j j}^{\lambda}= \begin{cases}1, & \text { se } j \in J \text { ed } d_{i j}-t \lambda_{i}<0 \\ 0, & \text { caso contrário }\end{cases}$

e para obter soluções aproximadas para o multiplicador lagrangeano/surrogate $t$, utiliza-se o seguinte algoritmo BM (busca do multiplicador):

- Defina o valor inicial $t_{0}$ e o tamanho do passo $s$;

- Faça $t \leftarrow t_{0}, a \leftarrow$ indefinido; $b \leftarrow$ indefinido;

- Enquanto ( $\mathrm{a}=$ indefinido ou $b=$ indefinido) repita:

- Resolva $L_{t} S_{\lambda} P P M$ e defina $x^{\lambda}$ conforme acima;

- Faça slope - $^{\lambda} \sum_{i \in N} \lambda_{i}\left(1-\sum_{j \in N} x_{i j}^{\lambda}\right)$;

- Se $\operatorname{slope}^{\lambda}>0$, faça $a \leftarrow t$ e $t \leftarrow t+s$;

- Senão, faça $b \leftarrow t$ e $t \leftarrow t-s$;

- Encontre o melhor valor de $t \in[\mathrm{a}, \mathrm{b}]$ pelo método da BISSEÇÃO.

Testes realizados em Pereira et al. (2005) comprovam que a obtenção de bons limitantes não dependem do cálculo exato de $t^{*}$, sendo suficiente encontrar um valor $t \in[a, b]$. O método da bisseção é utilizado neste momento e o seu algoritmo é utilizado da seguinte forma: deve-se encontrar $t_{m l}=(a+b) / 2$, que é a média aritmética entre $a$ e $b$, e este novo $t$ será utilizado para 
calcular os novos valores $x^{\lambda}$ e um novo slope (inclinação), se o slope for positivo deve-se substituir o valor de $a$ por $t_{m l}$ e caso contrário substitui-se o valor de b por $t_{m l}$. Com o valor atualizado de a ou de b, faz uma nova média aritmética encontrando $t_{m 2}$ e assim procede-se por $n$ iterações (neste trabalho usado vários valores para $n$ ), fixa-se $t^{*}$ como o valor que resultar no melhor valor de $L_{t} S_{\lambda} P P M$. Notar que o vetor $\lambda_{i}$ não muda dentro deste algoritmo, mudando apenas o valor de $t$ a cada iteração.

A mediana escolhida como centro do cluster de menor contribuição ao valor de $v\left(D_{t, \lambda}\right)$ corresponde ao vértice $j^{*}$ obtido como solução ótima do subproblema:

$S G C_{t}$

$$
v\left(S G C_{t}\right)=\underset{j \in N}{\operatorname{Min}}\left\{\underset{a_{i j} \in\{0,1\}}{\operatorname{Min}_{i \in N}} \sum_{i j}\left(d_{i j}-t \lambda_{i}\right) a_{i j}\right\}
$$

Considerando cada vértice $j \in N$ como mediana e fixando $a_{i j}$, $\forall i \in N$, o problema $S G C_{t}$ é resolvido, por inspeção, da seguinte maneira:

$$
a i j= \begin{cases}1, & \text { se } d i j-t \lambda i \leq 0 . \\ 0, & \text { se } d i j-t \lambda i>0 .\end{cases}
$$

Sendo $j^{*}$ o índice que resultar no menor valor para $\mathrm{v}\left(S G C_{t}\right)$, define-se um novo subconjunto $S_{j}^{*}$ como: $S_{j}^{*}=\left\{i \in N \mid a_{i j^{*}}=1\right\}$ e a coluna $A^{j^{*}}$ será incluída em $\overline{P C C}$ se:

$$
\sum_{i \in N}\left(d_{i j^{*}}-\lambda_{i}\right) a_{i j^{*}}<\mu
$$

Para acelerar o processo de resolução utiliza-se, neste momento, a estratégia de multipricing, ou seja, todas as colunas que satisfizerem a condição acima serão adicionadas ao $P M R$.

\subsubsection{A Retirada de Colunas}

Para obter um gerenciamento do tamanho do problema deve-se levar em consideração que a medida que ocorre a inclusão de novas colunas à formulação do $P M R$, este também será aumentado. Deste modo, pode-se exigir mais recursos computacionais que os disponíveis para sua solução. Assim, uma rotina para retiradas de colunas, que não contribuem significativamente para uma melhoria na solução, pode ser utilizada.

A remoção dessas colunas ocorre quando o problema apresentar um número de colunas maior que um valor estabelecido anteriormente e/ou sempre que se desejar eliminar da formulação as colunas com custo reduzido elevado, quando comparadas com um valor médio de referência.

Deve-se levar em conta que a utilização desse procedimento também pode ser prejudicial para o processo de resolução do problema, pois ao remover colunas pode-se retirar informações relevantes da formulação prejudicando a solução final. 
Um parâmetro fator_rc permite controlar a intensidade do teste aplicado para a remoção de colunas, limitando o número de colunas consideradas úteis para o problema. O fator_rc $\in(0,1)$ deve ser escolhido adequadamente pois, dependo da característica do problema, se o fator_rc for próximo de 0 (zero) então a retirada de colunas será em maior número, acelerando o processo mas prejudicando a solução final, e se esse fator_rc for próximo de 1(um) então o processo é relaxado para obtenção de uma melhor solução porém com um tempo médio maior de execução do algoritmo. A rotina utilizada é a seguinte:

- Defina TotC como o número total de colunas de $\overline{P C C}$;

- Defina fator_rc como um número positivo;

- Defina $c r$ médio como o custo reduzido médio das colunas do conjunto inicial;

- Obtenha $c r_{j}, j=1, \ldots, \operatorname{Tot} C$, o custo reduzido de cada coluna $j$ de $\overline{P C C}$;

- $\quad$ Para $\mathrm{j}=1, \ldots$, Tot $C$ faça

- $\operatorname{Se} c r_{j}>$ fator_rc $* c r \_m e ́ d i o$, remova a coluna $j$ de $\overline{P C C}$;

- Fim

\subsubsection{O Algoritmo de Geração de Colunas}

O algoritmo de geração de colunas é o seguinte:

- Faça condição $\leftarrow$ TRUE;

- Enquanto (condição = TRUE) faça

- Resolva $\overline{P C C}$ e obtenha também os valores ótimos das variáveis duais $\lambda_{i} \mathrm{e}$ $\mu$

- Obtenha uma solução aproximada para $D_{t, \lambda}$;

- Se $\left|v(\overline{P C C})-v\left(L_{t} S_{\lambda} P P M\right)\right|<\varepsilon$, faça condição $\leftarrow$ FALSE;

- Senão

- Execute testes para remoção de colunas e aplique a rotina de retiradas de colunas, se necessário;

- Resolva o problema $S G C_{t}$ e adicione à $\overline{P C C}$ as colunas $A^{j}$ que satisfazem a condição;

- Se não houver tais colunas faça condição $\leftarrow$ FALSE;

- Fim;

- Fim 
Se $t=1$, isto é, o caso lagrangeano tradicional, não é necessário resolver $D_{t, \lambda}$. Para a resolução de $\overline{P C C}$ pode-se utilizar um software comercial que possui ferramentas para a construção e resolução de modelos matemático de otimização como, por exemplo, LINGO (LINDO Systems inc.), CPLEX (ILOG inc.) e vários outros, respeitando suas características, ou ainda programar o método Simplex (revisado) e calcular a redução de custo das colunas, bem como as variáveis duais para o problema. Tanto para o caso lagrangeano tradicional ou para o caso de Lagrangeano/surrogate o conjunto inicial de colunas gerado será o mesmo.

\subsubsection{Regras de Ramificação}

Na ramificação da árvore, dois novos $P M R$ 's são criados e acrescidos na lista de modelos do algoritmo. O processo, chamado bifurcação, tem o efeito de contrair a região viável de um modo que elimina de considerações posteriores a solução corrente não inteira para o par de vértices $(q, r)$, preservando ainda todas as possíveis soluções inteiras do problema original. Considera-se que os ramos à esquerda, neste trabalho modelos $P$ com índice par, correspondem aos problemas nos quais os coeficientes das linhas $q$ e $r$ de uma dada coluna com valor fracionário na solução final do $P M R$ são idênticos, e que nos ramos à direita, modelos $P$ com índice ímpar, apenas um dos coeficientes pode assumir o valor 1. Esta regra permite identificar os pares de vértices que pertencem ao mesmo cluster na solução ótima de $\overline{P C C}$. O par de vértices $(q, r)$ que definirá a ramificação é determinado da forma descrita a seguir.

\section{Testes Computacionais}

Dentre os testes realizados neste trabalho, apresentam-se as três melhores novas propostas utilizando diferentes números de cidades-sede das divisões. Nas duas últimas propostas, mudam-se os critérios para escolha das cidades candidatas à sede de divisão, explicados posteriormente. A escolha das cidades-sede feita pelo algoritmo e conseqüentemente a construção do grupo de cidades, vinculadas a uma cidade-sede, leva em consideração além da distância, a capacidade de atendimento das cidades, restringindo as cidades candidatadas à sede de divisão de acordo com o número de procedimentos realizados pela cidade.

As macro-regiões na configuração atual tem como cidades-sede as 6 principais cidades do estado, na área de saúde pública: Cascavel, Curitiba, Maringá, Londrina, Pato Branco e Ponta 
Grossa. A configuração atual ainda possui 22 regionais e 83 micro-regiões. Os dados são todos reais e obtidos dos órgãos governamentais responsáveis.

Na primeira proposta, alterou-se a configuração atual apenas em relação ao número sedes de macro-região, aumentando para 7 cidades, incluindo a cidade de Paranavaí. Os critérios utilizados para escolher a cidade de Paranavaí são: sua localização privilegiada na região Noroeste do estado (região mais carente de atendimento) e a sua capacidade de atendimento ser equivalente às cidadessede já definidas. Após a construção das macro-regiões, determinam-se as 22 regionais, respeitando a proporcionalidade de cidades em cada macro-região, e por fim as 83 micro-regiões também considerando a proporção vigente.

A segunda e a terceira propostas são apresentadas a seguir, ambas com apenas cinco macroregiões, sendo as cidades-sede: Cascavel, Curitiba, Guarapuava, Londrina e Maringá. Os critérios usados para escolher a cidade de Guarapuava como sendo sede de macro-região ao invés de Pato Branco ou de Ponta Grossa são: excelente localização geográfica na região central do estado e a equivalência de capacidade de atendimento em relação às cidades retiradas.

$\mathrm{Na}$ segunda proposta escolhem-se apenas 18 cidades-sede de regionais entre as 40 principais cidades do estado, selecionadas pelo critério de capacidade de atendimento médico. Depois de determinadas através do algoritmo as regionais, determinam-se as 43 cidades-sede de micro-região, sendo esse número escolhido entre as 100 principais cidades do estado.

$\mathrm{Na}$ terceira proposta, aumentou-se para 22 as sedes de regionais, escolhidas agora entre as 50 cidades mais capacitadas para atendimento, já retiradas as cinco sedes de macro-região. As 43 sedes de micro-região foram determinadas da mesma forma da primeira proposta. Nesta proposta, porém, não se impõe a necessidade das cidades pertencentes a uma micro-região estarem na mesma regional, bem como as cidades de uma regional não necessitarem estar na mesma macro-região.

Desta forma, o algoritmo determina as divisões hierárquicas sem nenhum tipo de restrição, ou seja, são escolhidas sempre as cidades-sede de divisão entre todas as cidades candidatas, independente da divisão superior, e ainda se uma cidade é sede de uma divisão não poderá mais ser candidata à sede de uma divisão inferior.

Assim, cada cidade do estado terá sempre diferentes opções para seus cidadãos procurarem atendimento médico no caso em que o atendimento só pode ser feito pela própria cidade ou pela cidade-sede de sua divisão, como hoje se discute na secretaria estadual de saúde. Assim garante-se, nessa proposta discutida pela SESA-PR, opções adicionais para os cidadãos das cidades menos estruturadas.

As figuras 1 e 2 mostram os mapas das macro-regiões para comparação entre a situação atual e as três propostas. As figuras 3, 4 e 5 apresentam: as regionais da situação atual (diferenciadas por cores); as regionais (diferenciadas por cores) e as micro-regiões (diferenciadas 
por reforço das suas fronteiras) encontradas nas propostas realizadas. As micro-regiões atuais (figura 3) não são mostradas em um mapa devido ao fato de existir cidades pertencentes a mais de uma micro-região o que tornaria o mapa "poluído" e confuso. Os pontos brancos no mapa são as cidades-sede de regionais e os pontos pretos são as cidades-sede de micro-regiões, as cidades em branco são sedes em uma divisão superior.

Figura 1 - Mapa das macro-regiões atuais.

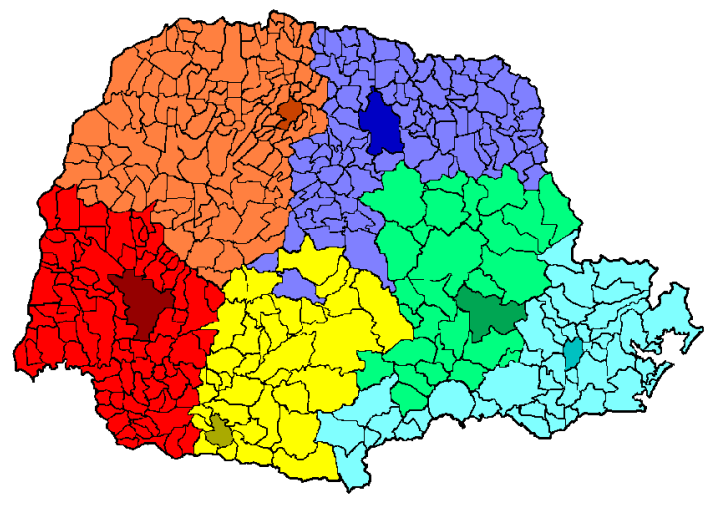

Figura 2 - Mapas das macro-regiões: proposta 1 (esquerda) x propostas 2 e 3 (direita).
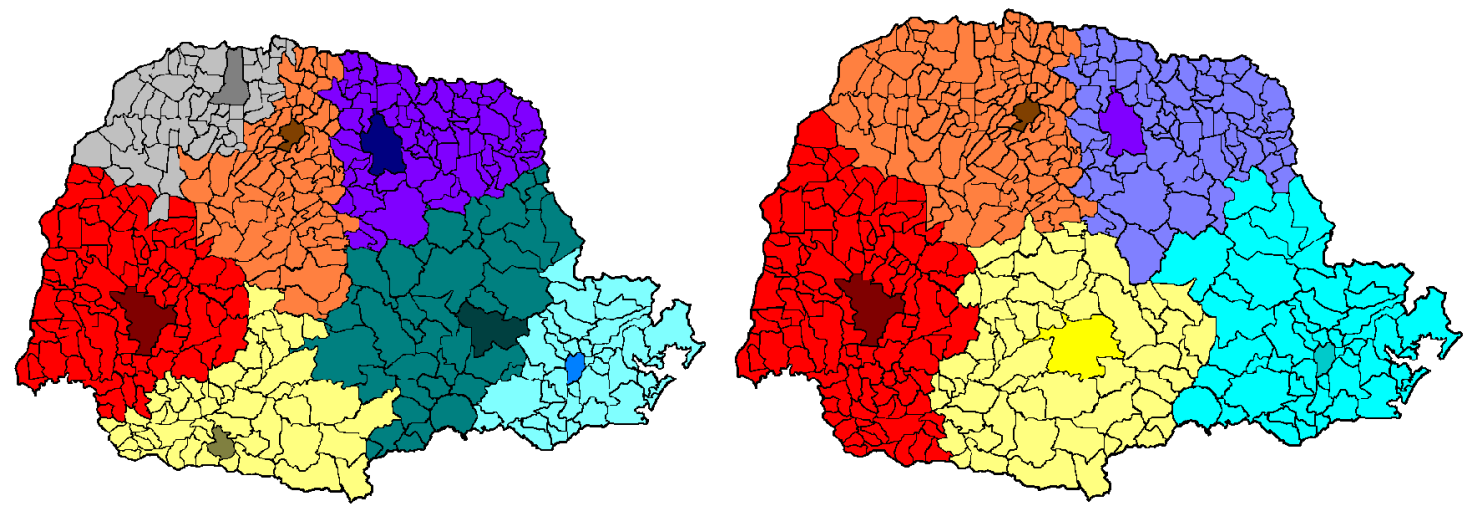

Figura 3 - Mapas das Regionais e micro-regiões: atual (esquerda) x proposta 1 (direita).
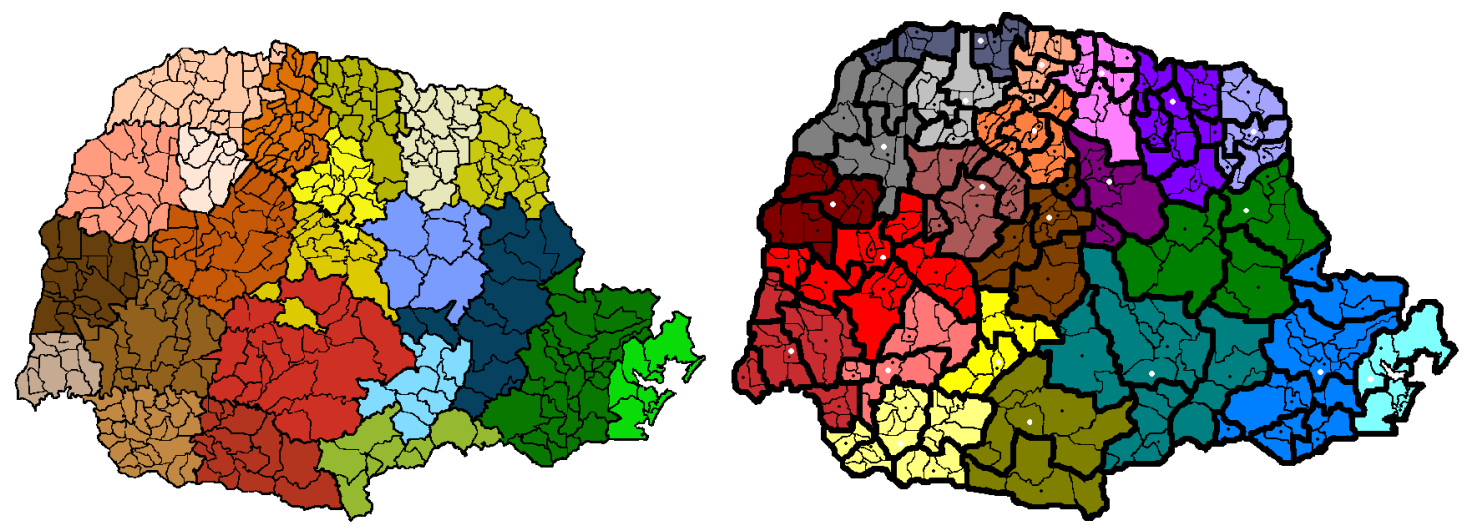
Figura 4 - Mapas das Regionais (esquerda) e micro-regiões (direita): proposta 2.
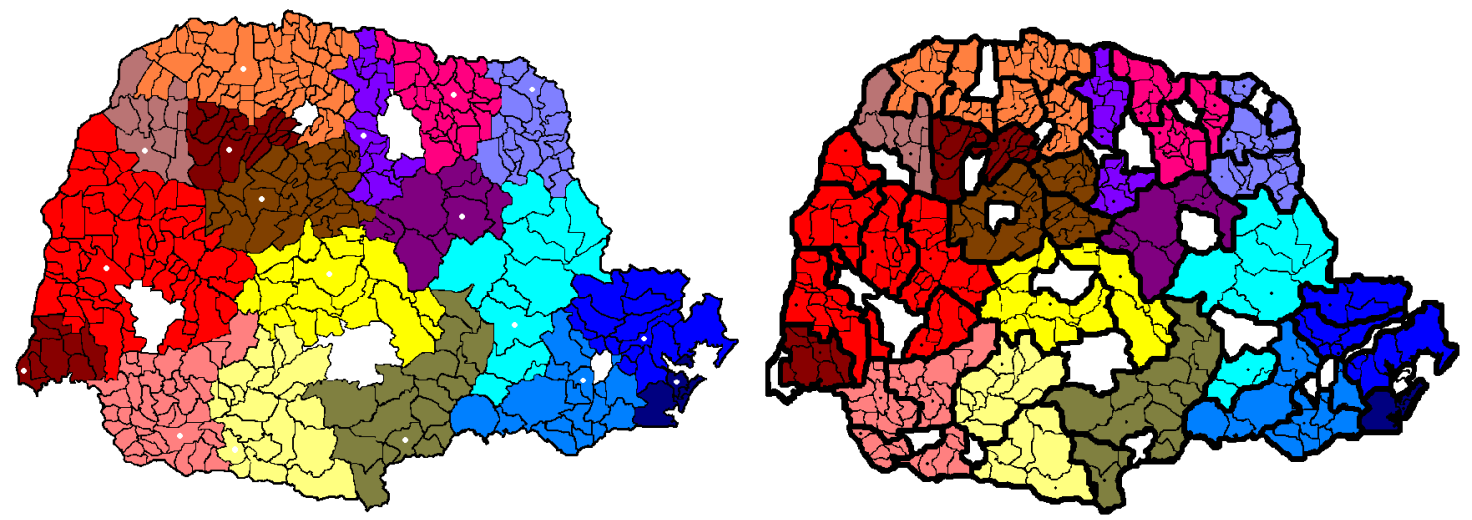

Figura 5 - Mapas das Regionais (esquerda) e micro-regiões (direita): proposta 3.
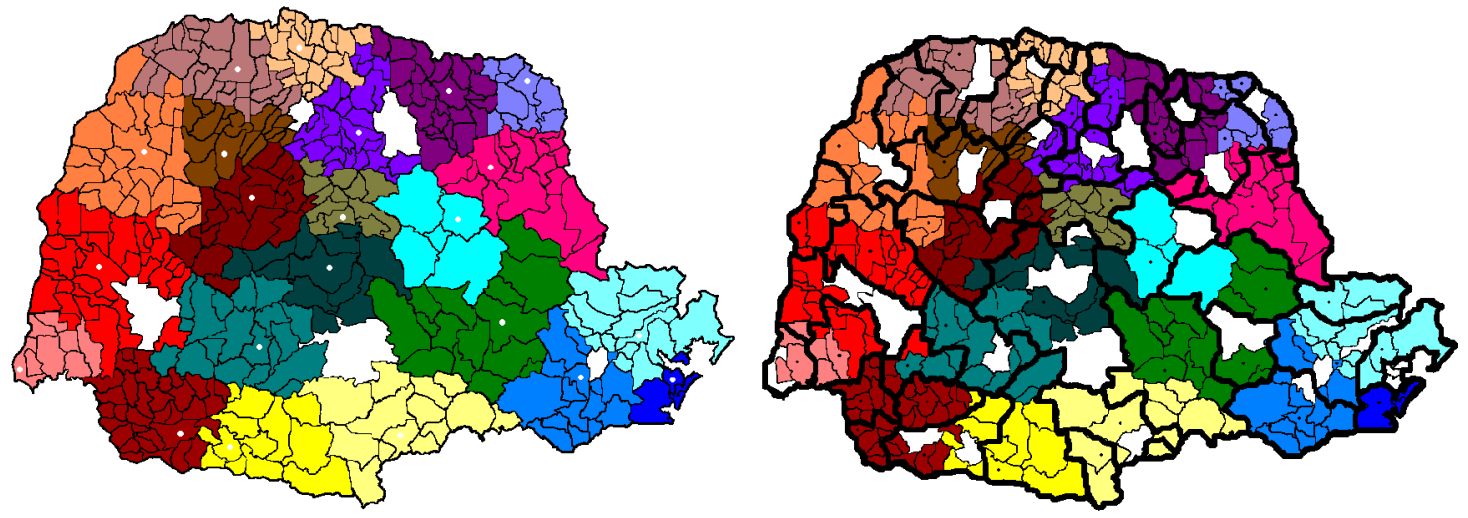

O quadro 1 apresenta os resultados numéricos da utilização do algoritmo, onde tem-se o somatório das distâncias de cada cidade até as suas respectivas cidades-sede de cada sub-divisão. O referido quadro apresenta, ainda, a diferença da configuração atual para cada teste por sub-divisão e no total.

Quadro 1 - Resultados comparando as distâncias entre as configurações.

\begin{tabular}{|c|ccc|ccc|c|}
\hline $\begin{array}{c}\text { Configurações } \\
\text { Da } \\
\text { Hierarquização }\end{array}$ & \multicolumn{3}{|c|}{$\begin{array}{c}\text { Somatório das } \\
\text { Distâncias }\end{array}$} & \multicolumn{3}{c|}{$\begin{array}{c}\text { Diferença em } \\
\text { Relação à Atual }\end{array}$} & \multirow{2}{*}{$\begin{array}{c}\text { Diferença } \\
\text { Total }\end{array}$} \\
\cline { 2 - 8 } & Macro & Regional & Micro & Macro & Regional & Micro & - \\
Atual & 45651 & 22574 & 14614 & - & - & - & - \\
Proposta 1 & 34126 & 18169 & 7725 & 11525 & 4405 & 6889 & 22819 \\
Proposta 2 & 38728 & 24910 & 10736 & 6923 & -2336 & 3878 & 8465 \\
Proposta 3 & 38728 & 18945 & 13632 & 6923 & 3629 & 982 & 11534 \\
\hline
\end{tabular}

\section{Conclusões}

Neste trabalho foi utilizada a técnica de geração de colunas no algoritmo branch-and-price para encontrar novas configurações na divisão hierárquica das cidades do estado do Paraná. Vale salientar que neste trabalho não foi feita a comparação do método branch-and-price com outros métodos heurísticos e/ou meta-heurísticos, ficando este ponto como sugestão para trabalhos futuros. 
Um ponto importante nas duas últimas propostas é o fato de possuírem menos divisões hierárquicas do que a atual, principalmente nas micro-regiões, e a distância total da configuração se equivaler à atual. A conseqüência deste fato é que o investimento em saúde pública se torna mais eficaz, equilibrando a capacidade das cidades-sede para atendimento.

Os resultados mostram que existem melhores possibilidades para a divisão hierárquica entre as cidades no estado do Paraná e que a utilização do algoritmo branch-and-price pode ser uma ferramenta de grande valia para se encontrar medianas em problemas considerados $N P$-hard. Com uma divisão hierárquica otimizada, uma metodologia de investimento mais econômico para a secretaria de saúde do estado pode ser desenvolvida.

Ao se definir divisões hierárquicas otimizadas pode-se investir a verba destinada à saúde respeitando essa divisão, isto é, melhorando os recursos médicos das cidades-sede de forma igualitária. Assim, a medida que a verba seja disponibilizada, verifica-se qual divisão está mais defasada em relação às demais de mesmo grau hierárquico e investe-se nela, tentando homogeneizar as capacidades de atendimento das cidades-sede.

Desta forma, com o passar do tempo, poder-se-á encontrar as micro-regiões com capacidade para atendimento dos procedimentos de baixa complexidade, as cidades-sede de regionais poderão atender em sua totalidade os procedimentos médicos de média complexidade e ter-se-á seis cidadessede de macro-regiões com capacidade de atender todos os pacientes de sua grande região que necessitem de atendimento de alta complexidade.

\section{Referências}

BRASIL. Ministério da Saúde. Secretaria de Assistência à Saúde. Regionalização da assistência à saúde: aprofundando a descentralização com eqüidade no acesso. Departamento de Descentralização da Gestão da Assistência. Norma Operacional da Assistência à Saúde - NOAS-SUS 01/02 (Portaria MS/GM n. ${ }^{\circ}$ 373, de 27 de fevereiro de 2002 , e regulamentação complementar). 2. ${ }^{a}$ edição revista e atualizada. Brasília/DF: Ministério da Saúde, 2002.

BRASIL. Conselho Nacional de Secretários da Saúde. Regionalização solidária: proposta para a Oficina Agenda para um novo pacto de gestão do SUS. Brasília: CONASEMS, 2004. Documento preliminar.

NARCISO, M. G. A relaxação lagrangeana/surrogate e algumas aplicações em otimização combinatória. 1998. 134p. Tese (Doutorado em Computação Aplicada) Instituto Nacional de Pesquisas Espaciais, São José dos Campos. 1998.

OLIVEIRA, E. Redes e regionalização da saúde. IBGE. Ceará, 2005.

PARANÁ. Secretaria de Estado da Saúde. Plano diretor de regionalização. Curitiba, 2001.

PEREIRA, M. A.; LORENA, L. A. N.; SENNE, E. L. F. Um método Branch-and-price para o problema de localização de p-medianas. São José dos Campos. 2005. 88 p. Tese (Doutorado em Computação Aplicada) - Instituto Nacional de Pesquisas Espaciais.

RAO, M. R. Cluster analysis and mathematical programming. Journal of the American Statistical Association, v. 66 , p. 622-626, 1971. 
RIO GRANDE DO NORTE. Plano diretor de regionalização. Governo do Estado do Rio Grande do Norte. Secretaria de Estado da Saúde Pública. Coordenadoria de Planejamento e Controle do Sistema de Saúde - CPCS, 2004.

ROSÁRIO, R. R. L.; CARNIERI, C; STEINER, M. T. A. Proposta de solução para o problema das p-Medianas na localização de unidades de saúde 24 horas. 119 p. Curitiba-PR, 2002. Dissertação de Mestrado, CPGMNE - UFPR.

SCARPIN, C. T.; STEINER, M. T. A.; DIAS, G. J. C. Técnicas da Pesquisa Operacional Aplicadas na Otimização do Fluxo de Pacientes do Sistema Único de Saúde do Estado do Paraná. XXXVIII SBPO - SIMPÓSIO BRASILEIRO DE PESQUISA OPERACIONAL. Anais. Goiânia (GO), Brasil, 2006.

\begin{abstract}
This paper presents three news proposals for optimizing the health system in the state of Paraná. The project consists in dividing the state into smaller regions, in three hierarchic levels: macroregions, regional and micron-regions, made up by several cities assigned to a regional seat in charge of major procedures at its resolution level. The optimized formation of groups of cities is the main objective of this work. The classical model that is used to represent problems in this class is the pmedians problem, which searches for $p$ points (medians) in a n-node network, considering them as the facilities and trying to minimize the sum of the distances from the $(n-p)$ demand points to the $p$ medians. To solve this problem the branch-and-price method was used, based on the traditional branch-and-bound, by applying to each node in the tree the column generating method that uses the lagrangean/surrogate relaxation as a stabilization alternative. The results obtained were considered very consistent.
\end{abstract}

Key-words: hierarchical configurations in the health system; p-medians problem; branch-and-price algorithm.

\title{
Inserir aqui dados completos de TODOS os autores:
}

Nome completo: Cassius Tadeu Scarpin

Filiação institucional: Universidade Federal do Paraná

Departamento: PPGMNE

Função ou cargo ocupado: Doutorando

Endereço completo para correspondência (bairro, cidade, estado, país e CEP): Rua Professor João

Soares Barcelos, 80 A, Hauer, Curitiba, Paraná, Brasil, CEP 81630-060.

Telefones para contato: (41) 30397239 / (41) 91072942

e-mail: cassiusts@yahoo.com.br

Nome completo: Maria Teresinha Arns Steiner

Filiação institucional: Universidade Federal do Paraná

Departamento: Matemática

Função ou cargo ocupado: Professora Titular

Endereço completo para correspondência (bairro, cidade, estado, país e CEP): Centro Politécnico

s/n - Bloco Lame/Cesec, Jd Américas, Curitiba, Paraná, Brasil, CEP 81531-990.

Telefones para contato: (41) 33613218 
e-mail: tere@ufpr.com.br

Nome completo: Gláucio José Cardozo Dias

Filiação institucional: Universidade Federal do Paraná

Departamento: PPGMNE

Função ou cargo ocupado: Mestrando

Endereço completo para correspondência (bairro, cidade, estado, país e CEP): Centro Politécnico s/n - Bloco Lame/Cesec, Jd Américas, Curitiba, Paraná, Brasil, CEP 81531-990.

Telefones para contato: (41) 33613218

e-mail: glaucio.dias@datasul.com.br 